University of Nebraska - Lincoln

DigitalCommons@University of Nebraska - Lincoln

Faculty Publications in Food Science and Technology

$2-2008$

Repression of Proinflammatory Gene Expression by Lipid Extract of Nostoc commune var sphaeroides Kützing, a Blue-green Alga, via Inhibition of Nuclear Factor-kB in RAW 264.7 Macrophages

\author{
Young-Ki Park \\ University of Nebraska-Lincoln \\ Heather E. Rasmussen \\ University of Nebraska-Lincoln, heather.rasmussen@unl.edu \\ Sarah J. Ehlers \\ University of Nebraska-Lincoln \\ Kara R. Blobaum \\ University of Nebraska-Lincoln \\ Fan Lu \\ Algaen Corporation
}

See next page for additional authors

Follow this and additional works at: https://digitalcommons.unl.edu/foodsciefacpub

Part of the Food Science Commons

Park, Young-Ki; Rasmussen, Heather E.; Ehlers, Sarah J.; Blobaum, Kara R.; Lu, Fan; Schlegel, Vicki L.; Carr, Timothy P.; and Lee, Ji-Young, "Repression of Proinflammatory Gene Expression by Lipid Extract of Nostoc commune var sphaeroides Kützing, a Blue-green Alga, via Inhibition of Nuclear Factor-KB in RAW 264.7 Macrophages" (2008). Faculty Publications in Food Science and Technology. 270.

https://digitalcommons.unl.edu/foodsciefacpub/270

This Article is brought to you for free and open access by the Food Science and Technology Department at DigitalCommons@University of Nebraska - Lincoln. It has been accepted for inclusion in Faculty Publications in Food Science and Technology by an authorized administrator of DigitalCommons@University of Nebraska Lincoln. 


\section{Authors}

Young-Ki Park, Heather E. Rasmussen, Sarah J. Ehlers, Kara R. Blobaum, Fan Lu, Vicki L. Schlegel, Timothy

P. Carr, and Ji-Young Lee 
Published in Nutrition Research 28:2 (February 2008), pp. 83-91; doi: 10.1016/j.nutres.2007.11.008 Copyright (C) 2008 Elsevier, Inc. Used by permission.

Submitted July 19, 2007; revised November 19, 2007; accepted November 21, 2007; published online January 26, 2008.

\title{
Repression of Proinflammatory Gene Expression by Lipid Extract of Nostoc commune var sphaeroides Kützing, a Blue-green Alga, via Inhibition of Nuclear Factor- $\kappa$ B in RAW 264.7 Macrophages
}

\author{
Young-Ki Park, ${ }^{1}$ Heather E. Rasmussen, ${ }^{1}$ Sarah J. Ehlers, ${ }^{1}$ \\ Kara R. Blobaum, ${ }^{1}$ Fan Lu, ${ }^{2}$ Vicki L. Schlegal, ${ }^{3}$ Timothy P. Carr, ${ }^{1}$ \\ and Ji-Young Lee ${ }^{1}$
}

1. Department of Nutrition and Health Sciences, University of Nebraska-Lincoln, Lincoln, Nebraska, USA

2. Algaen Corporation, Winston Salem, North Carolina, USA

3. Department of Food Science and Technology, University of Nebraska-Lincoln, Lincoln, Nebraska, USA

Corresponding author - Ji-Young Lee, phone 402-472-3816, fax 402-472-1587, email jlee8@unl.edu

\begin{abstract}
We investigated whether lipid extract from a blue-green alga, N. commune, modulates proinflammatory gene expression in RAW 264.7 macrophages. The cells were incubated with $N$. commune lipid extract $(0-100 \mu \mathrm{g} / \mathrm{mL})$ and subsequently activated by LPS (100 ng/mL). Quantitative real-time PCR analysis showed that mRNA abundance of proinflammatory mediators, including TNF- $\alpha$, COX-2, IL-1 $\beta$, IL-6, and iNOS, was significantly reduced by $N$. commune lipid extract in a dose-dependent manner. Secretion of TNF- $\alpha$ and IL-1 $\beta$ into cell culture medium was also significantly decreased by N. commune lipid extract. Thin-layer chromatography-densitometry analysis showed that N. commune lipid extract contained approximately $15 \%$ of fatty acids. To determine whether the inhibition
\end{abstract}


of proinflammatory mediator production by $N$. commune lipid extract is primarily conferred by fatty acids in the lipid extract, macrophages were incubated with $100 \mu \mathrm{g} / \mathrm{mL}$ of $N$. commune lipid extract or $15 \mu \mathrm{g} / \mathrm{mL}$ of a fatty acid mixture, which was formulated to reflect the fatty acid composition of $N$. commune lipid extract. The fatty acid mixture significantly reduced RNA abundance of TNF- $\alpha$ and COX-2, but to a lesser extent than did the N. commune lipid extract, suggesting the presence of additional bioactive compounds with an anti-inflammatory property in the lipid extract. As NF- $\kappa \mathrm{B}$ is a major regulator for the proinflammatory gene expression, we measured its DNA-binding activity. DNA-binding activity of NF- $\kappa \mathrm{B}$ was significantly reduced by N. commune lipid extract. In conclusion, our study suggests that $N$. commune lipid extract represses the expression of proinflammatory genes in RAW 264.7 macrophages, at least in part, by inhibiting the activation of NF- $\kappa$ B pathway.

Keywords: blue-green algae, Nostoc commune var sphaeroides, anti-inflammatory, NF- $\kappa$ B, RAW 264.7 macrophages

Abbreviations: COX-2, cyclo-oxygenase 2; iNOS, inducible nitric oxide synthase; IL-1 $\beta$, interleukin$1 \beta$; IL-6, interleukin-6, LPS, lipopolysaccharide; NF- $\kappa \mathrm{B}$, nuclear factor-kappa B; N. commune, Nostoc commune var sphaeroides Kützing; TNF- $\alpha$, tumor necrosis factor- $\alpha$

\section{Introduction}

Chronic inflammation can result in an elevated risk of various chronic inflammatory diseases such as atherosclerosis, rheumatoid arthritis, chronic obstructive pulmonary disease, asthma, and inflammatory bowel disease. Pharmaceutical anti-inflammatory drugs are generally used to treat these diseases. In general, the anti-inflammatory drugs reduce inflammatory response by suppressing the production of proinflammatory mediators, such as tumor necrosis factor $\alpha$ (TNF- $\alpha$ ), interleukin $1 \beta$ (IL- $1 \beta)$, and nitric oxide, which are involved in the pathogenesis of inflammatory diseases [1,2]. However, reports on the increased risk of cardiovascular disease with long-term use of several nonsteroidal antiinflammatory drugs have raised concerns over using the drugs [3]. In addition, increasing the public's preference for natural and herbal medicines to drug therapies in preventing and treating chronic inflammatory diseases has prompted the discovery and development of new bioactive natural products with anti-inflammatory properties.

Blue-green algae, also termed cyanobacteria, are among the most primitive forms of life on Earth [4]. Bioactive compounds isolated from blue-green algae have been shown to be neuroprotective, cytotoxic, antibacterial, antifungal, antiviral, and anti-inflammatory [5-9]. Edible blue-green algae, such as Spirulina and Aphanizomenon flos-aquae, are currently marketed as dietary supplements with various health claims for immune function, inflammation, heart disease, and general well-being. Concerns over naturally grown blue-green algae, however, have been raised because of contamination from algal toxins and heavy metals. During the harvesting of natural blue-green algae, they are easily contaminated with toxin-producing algae such as Microcystis aeruginosa, which generates microcystins, potent hepatotoxins [10]. In addition, the quality of naturally grown blue-green algae can vary depending on the growth environments; for example, altitude, temperature, and sun exposure [11]. 
To develop a new natural source with health-enhancing properties, an edible blue-green alga, Nostoc commune var sphaeroides Kützing (N. commune), was cultivated. N. commune has been used as a food delicacy or herbal medicine in Asian, African, and South American countries for centuries [12]. N. commune has been used as an ingredient of Chinese medicine since the Eastern Jin Dynasty (317-420 AD) as recorded in The Supplement to Compendium of Materia Medica [13]. It has been historically suggested that N. commune can treat a variety of medical conditions, including inflammation, night blindness, digestion, burns, anxiety, indigestion, and chronic fatigue. In addition, a hypocholesterolemic effect of $N$. commune was suggested in rats fed N. commune, which was attributed to its high content of dietary fibers [14]. Although studies have also demonstrated various health benefits from $N$. commune, including cholesterol lowering, antiviral, anticancer, and anti-inflammatory activities [8,14-19], a mechanistic investigation on the biological functions of N. commune is very limited.

In the present study, we intended to elucidate the molecular mechanisms responsible for an anti-inflammatory function of cultivated N. commune, which is free of contamination of toxins and whose quality can be controlled by culture conditions. Our hypothesis was that lipid extract of $N$. commune exerts an anti-inflammatory property by repressing the expression of proinflammatory genes through the inhibition of nuclear factor- $\kappa \mathrm{B}(\mathrm{NF}-\kappa \mathrm{B})$, a major transcription factor for proinflammatory response. As macrophages play an important role in the innate immune system, we performed our experiments using murine RAW 264.7 macrophages, as the macrophages are widely used as an in vitro model for studies on inflammatory pathways. Our data indicate that lipid extract of N. commune reduced the production of proinflammatory mediators, such as TNF- $\alpha$, IL- $1 \beta$, cyclooxygenase 2 (COX-2), inducible nitric oxide synthase (iNOS), and IL-6. This repression is mediated, at least in part, by inhibiting the activation of NF- $\kappa$ B. Our study provides, for the first time, scientific support for $N$. commune to be developed as a nutritional food to reduce the risk of inflammatory diseases.

\section{Methods and materials}

\subsection{Preparation of $\mathrm{N}$. commune lipid extract}

Fresh N. commune was obtained from Algaen Corporation (Winston Salem, North Carolina). N. commune was freeze-dried and ground for lipid extraction using the Bligh-Dyer method [20]. Briefly, $42 \mathrm{~mL}$ of chloroform/methanol (1:2 vol/vol) was added to approximately $3 \mathrm{~g}$ of dry $\mathrm{N}$. commune and vortexed. After the addition of $14 \mathrm{~mL}$ of chloroform and $10.6 \mathrm{~mL}$ of distilled water, the sample was mixed and filtered using a Whatman filter no. 42 (Whatman, Inc., Ann Arbor, Michigan) to remove solids. Subsequently, $6.3 \mathrm{~mL}$ of $0.05 \%$ $\mathrm{H}_{2} \mathrm{SO}_{4}$ was added, and samples were vortexed. To separate phases, samples were centrifuged at $500 \times g$ for 5 minutes and the lower liquid phase was recovered. Lower phase was dried to completion under nitrogen, weighed for lipid mass, and resuspended in chloroform. Tubes containing lipid extract were purged with nitrogen and tightly sealed. The lipid extract was stored at $-80^{\circ} \mathrm{C}$ with an oxygen absorber Ageless (Mitsubishi Gas Chemical America, New York, New York) to prevent oxidation until use. To incorporate the lipid 
extract into cell culture medium, chloroform was evaporated under nitrogen, and medium was added for subsequent sonication at room temperature for 5 minutes.

\subsection{Compositional analysis of $\mathbf{N}$. commune lipid extract}

The simple lipids of $N$. commune lipid extract were determined by thin-layer chromatography (TLC)-densitometry using a solvent system of hexane/diethyl ester/acetic acid (85:15:2 by volume) as previously described [21]. Images of the plates were obtained with a Kodak Gel Logic 440 imaging system and Kodak ID image analysis software (Kodak, Rochester, New York), and the sample spots were compared against external standards for migration and net band intensity.

Fatty acid composition of $N$. commune lipid extract was determined by gas chromatography. An aliquot of N. commune lipid extract ( $2 \mathrm{mg}$ in chloroform) was dried under nitrogen. Fatty acid methyl esters were prepared by the method of Metcalfe et al [22] and quantified using a $0.25 \mathrm{~mm} \times 100 \mathrm{~m}$ CP-Sil-88 capillary column (Chrompack, Raritan, New Jersey) under the following conditions: initial temperature, $180^{\circ} \mathrm{C}$ for 20 minutes, increased to $250^{\circ} \mathrm{C}$ at $5^{\circ} \mathrm{C} / \mathrm{min}$; injector temperature, $270^{\circ} \mathrm{C}$; flame ionization detector temperature, $300^{\circ} \mathrm{C}$; helium carrier gas; and split ratio of 50:1.

The carotenoid content of lipid extract was determined by high-performance liquid chromatography as previously described [23].

\subsection{Cell culture and treatment}

Murine RAW 264.7 macrophages (ATCC no. TIB-71) were maintained in RPMI 1640 containing $10 \%$ FBS, $100 \mathrm{U} / \mathrm{mL}$ of penicillin, $100 \mu \mathrm{g} / \mathrm{mL}$ of streptomycin, $2 \times$ vitamins (from $100 \times$ vitamin solution, MediaTech, Herndon, Virginia), and $2 \mathrm{mmol} / \mathrm{L}$ L-glutamine in a humidified chamber at $37^{\circ} \mathrm{C}$ with $5 \%$ carbon dioxide. All cell culture supplies were purchased from MediaTech.

Cells were plated at a density of $6 \times 10^{5} \mu \mathrm{g}$ cells in a well of 12 -well plates. When cells became approximately $90 \%$ confluent, increasing concentrations of N. commune lipid extract $(0-100 \mu \mathrm{g} / \mathrm{mL})$ were added to culture medium for 12 hours, after which $100 \mathrm{ng} / \mathrm{mL}$ of lipopolysaccharide (LPS) (Salmonella enterica serotype typhimurium; Sigma-Aldrich, St. Louis, Missouri) was added to the cell medium to activate cells for 18 hours. In separate experiments, cells were incubated with either $N$. commune lipid extract $(100 \mu \mathrm{g} / \mathrm{mL})$ or a fatty acid mixture $(15 \mu \mathrm{g} / \mathrm{mL})$, consisting of $26 \%$ palmitic acid, $24 \%$ palmitoleic acid, $7 \%$ oleic acid, 16\% linoleic acid, and 25\% linolenic acid, for 12 hours. After the incubation, LPS ( $100 \mathrm{ng} / \mathrm{mL}$ ) was added to activate macrophages for 18 hours. Fatty acids were complexed with bovine serum albumin (BSA, approximate molar ratio of BSA/fatty acid $=1: 2.5$ ), and therefore, the same amount of BSA was added to all the treatments. For all cell culture experiments, cells that were not incubated with $N$. commune lipid extract served as a control.

\subsection{Cytotoxicity measurement}

Cytotoxicity of N. commune lipid extract was determined using In Cytotox crystal violet dye elution (CVDE) kit (Aniara, Mason, Ohio) according to the manufacturer's instructions. In brief, RAW 264.7 macrophages were plated at a density of 25,000 cells per well in 
a 96-well plate and incubated for 24 hours, after which 0 to $200 \mu \mathrm{g} / \mathrm{mL}$ of $N$. commune lipid extract was added for 24 hours. Cells were washed twice with $200 \mu \mathrm{L}$ per well of wash solution CVDE I and $100 \mu \mathrm{L}$ of labeling solution CVDE II was added to each well. After incubation for 10 minutes at room temperature, the cells were washed 4 times with distilled water. The plate was air dried, and the cell layer was dissolved with $100 \mu \mathrm{L}$ per well of solubilization solution CVDE III. The absorbance was measured at $540 \mathrm{~nm}$. Sodium dodecyl sulfate (SDS; $0.5 \mathrm{mmol} / \mathrm{L}$ ) was used as a positive control for cytotoxicity. Data are expressed as viability (\%) relative to control that was not incubated with $N$. commune lipid extract.

\subsection{Total RNA isolation and quantitative real-time PCR}

Cells were washed twice with cold phosphate buffered saline (PBS) and $1 \mathrm{~mL}$ of Trizol reagent (Invitrogen, Carlsbad, California) was added to each well of a 12-well plate to isolate total RNA following the manufacturer's protocol. One microgram of total RNA was treated with DNase I (Promega, Madison, Wisconsin) to remove genomic DNA contamination, and subsequently, RNA samples were reverse transcribed by MMLV reverse transcriptase (Promega, Madison, Wisconsin). Realtime PCR analysis was performed using the Sybr Green procedure and an ABI 7300 instrument (Applied Biosystems, Foster City, California). Primers for TNF- $\alpha$, COX-2, IL-1 $\beta$, IL-6, iNOS, and glyceraldehyde-3-phosphate dehydrogenase (GAPDH) were designed according to GenBank database using the Primer Express 3.0 software provided by ABI and listed in Table 1. Expression of mRNA values was calculated using the threshold cycle $(\mathrm{Ct})$ value, that is, the number of PCR cycles at which the fluorescent signal during the PCR reaches a fixed threshold. For each sample,

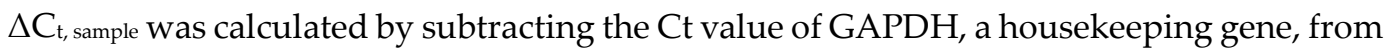
that of each gene of interest to normalize the data. The expression levels relative to control were estimated by calculating $\Delta \Delta \mathrm{C}_{\mathrm{t}}\left(\Delta \mathrm{C}_{\mathrm{t}}\right.$, sample $-\Delta \mathrm{C}_{\mathrm{t}}$, control $)$ and subsequently using the $2^{-\Delta \Delta \mathrm{Ct}}$ method [24].

\begin{tabular}{lll}
\hline \multicolumn{2}{l}{ Table 1. Real-time PCR primers } & \\
\hline Gene & Forward primer & Reverse primer \\
\hline TNF- $\alpha$ & 5'-GGCTGCCCCGACTACGT-3' & 5'-ACTTTCTCCTGGTATGAGATAGCAAAT-3' \\
IL-1 $\beta$ & 5'-GTCACAAGAAACCATGGCACAT-3' $^{\prime}$ & 5'-GCCCATCAGAGGCAAGGA-3' \\
COX-2 & 5'-AAAGGTTCTTCTACGGAGAGAGTTCA-3' & 5'-TGGGCAAAGAATGCAAACATC-3' \\
IL-6 & 5'-CTGCAAGAGACTTCCATCCAGTT-3' & 5'-AGGGAAGGCCGTGGTTGT-3' \\
iNOS & 5'-GCAGCTGGGCTGTACAAA-3' & 5'-AGCGTTTCGGGATCTGAAT-3' \\
GAPDH & 5'-TGTGTCCGTCGTGGATCTGA-3' & 5'-CCTGCTTCACCACCTTCTTGAT-3' \\
\hline
\end{tabular}

\subsection{Enzyme-linked immunosorbent assay for cytokines}

Concentrations of TNF- $\alpha$ and IL- $1 \beta$ in cell medium were determined by commercial mouse enzyme-linked immunosorbent assay (ELISA) kits (eBiosciences, San Diego, California) following the manufacturer's protocols. 


\subsection{Nuclear extract preparation and NF- $\kappa B$ activity measurement}

RAW 264.7 macrophages were incubated without or with N. commune lipid extract (100 $\mu \mathrm{g} / \mathrm{mL}$ ) followed by activation with $100 \mathrm{ng} / \mathrm{mL}$ of LPS for 1 hour. Cells were then washed twice with cold PBS, and nuclear fraction was prepared using Nuclear Extract kit (Active Motif, Carlsbad, California) following the manufacturer's instruction. Protein concentrations of samples were measured by BCA assay (Pierce, Rockford, Illinois) using BSA as a standard.

DNA-binding activity of p65 was assessed using an ELISA-based TransAM NF- $\kappa$ B p65 assay kit (Active Motif). Briefly, $20 \mu \mathrm{L}$ of complete lysis buffer containing $3 \mu \mathrm{g}$ of nuclear extract was added to a well of a 96-well plate coated with immobilized NF- $\kappa$ B consensus oligonucleotides and incubated for 1 hour with slow agitation. Primary NF- $\kappa$ B antibody was added and incubated for 1 hour, after which wells were washed 3 times with a washing buffer. Horseradish peroxidase-conjugated secondary antibody was added, and after 1-hour incubation, wells were washed 4 times with a washing buffer. Color was developed and optical density was measured by a spectrophotometer (Bio-Tek, Winooski, Vermont) at $450 \mathrm{~nm}$ with a reference wavelength of $655 \mathrm{~nm}$. Positive and negative controls were run simultaneously to validate the assay.

\subsection{Statistical analysis}

Treatment effects were analyzed by one-way analysis of variance (one-way ANOVA), and statistically significant differences among treatments were identified using Tukey's pairwise comparison using GraphPad InStat 3 (GraphPad Software, Inc., San Diego, California). Differences were considered significant at $P<.05$. Data are expressed as means \pm SEM.

\section{Results}

\subsection{Lipid composition of $\mathrm{N}$. commune lipid extract}

Extractable lipid compounds present in N. commune were determined by TLC-densitometry. The lipid extract contained pigments, free fatty acids, triacylglycerol, vitamin E, and wax esters/steryl esters (Fig. 1). Although a large percentage of pigments were observed in this lipid extract, identification of the pigments was beyond the scope of this study. The fatty acid composition of lipid extract was approximately $26 \%$ palmitic acid (16:0), $24 \%$ palmitoleic acid (16:1), 16\% linoleic acid (18:2), and 25\% linolenic acid (18:3) (Table 2). Assuming that carotenoids contributed to the high level of pigmentation in the lipid extract, we attempted to quantify several carotenoids, including $\alpha$-carotene, $\beta$-carotene, lutein, lycopene, cryptoxanthin, and zeaxanthin. Only $\beta$-carotene was detected with a concentration of $70 \mathrm{mg} / 100 \mathrm{~g}$ dry weight. 


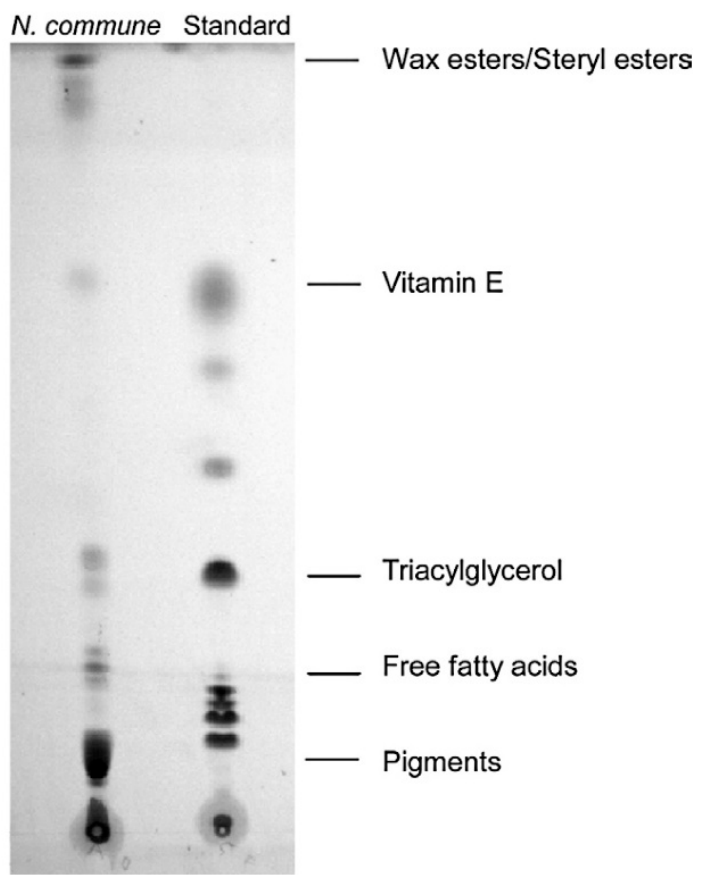

Figure 1. Lipid analysis of N. commune lipid extract by TLC-densitometry. Lipids of N. commune and lipid standards were fractionated on a silica gel plate and developed using hexane-diethyl ether-acetic acid ( $85: 15: 2$, by volume). Bands were visualized by submerging the plate in a solution of $10 \%$ cupric sulfate and $8 \%$ phosphoric acid and subsequently by charring the dried plate at $165^{\circ} \mathrm{C}$ for 10 minutes. The TLC standard for lipids is shown in the right.

\begin{tabular}{lc}
\hline Table 2. Fatty acid composition of $N$. commune lipid extract \\
\hline Fatty acid & \% of total fatty acids \\
\hline $14: 0$ & $0.62 \pm 0.02$ \\
$16: 0$ & $25.7 \pm 0.38$ \\
$16: 1 \Delta 9$ & $24.1 \pm 0.40$ \\
$18: 0$ & $2.2 \pm 0.23$ \\
$18: 1 \Delta 9$ & $4.3 \pm 0.16$ \\
$18: 1 \Delta 11$ & $2.2 \pm 0.03$ \\
$18: 2$ & $15.6 \pm 0.18$ \\
$18: 3$ & $25.3 \pm 0.18$ \\
\hline
\end{tabular}

Values are mean $\pm \operatorname{SEM}(n=6)$.

a. Fatty acid composition of N. commune lipid extract was determined by GC analysis.

\subsection{Cytotoxicity of $\mathrm{N}$. commune lipid extract in RAW 264.7 macrophages}

Cytotoxicity of N. commune lipid extract was assessed by incubating RAW 264.7 macrophages with increasing concentrations $(0-200 \mu \mathrm{g} / \mathrm{mL})$ of the lipid extract for 24 hours. Cells incubated with 25 to $200 \mu \mathrm{g} / \mathrm{mL}$ of $N$. commune lipid extract showed a comparable viability 
with control (Fig. 2). This result indicates that $N$. commune lipid extract is not cytotoxic to RAW 264.7 macrophages within this range.

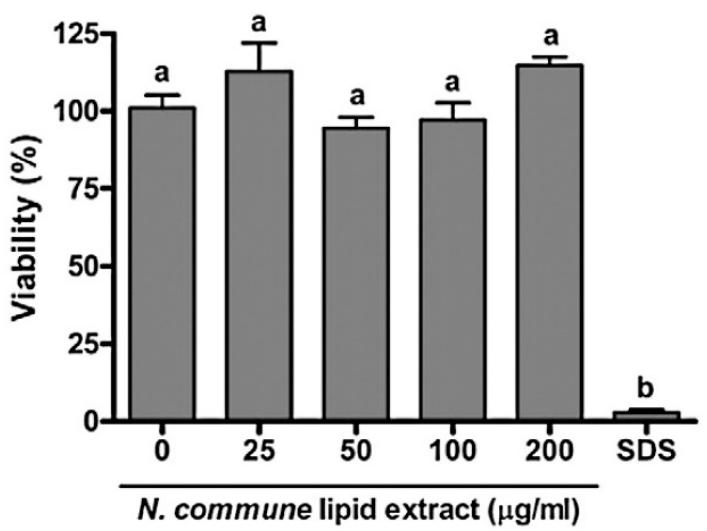

Figure 2. Cytotoxicity of N. commune lipid extract. RAW 264.7 macrophages were incubated with 0 to $200 \mu \mathrm{g} / \mathrm{mL}$ of $N$. commune lipid extract or $0.5 \mathrm{mmol} / \mathrm{L}$ SDS as a positive control for cytotoxicity for 24 hours. Cell viability was determined using In Cytotox crystal violet dye elution kit. Data are expressed as viability (\%) relative to control that was not incubated with $N$. commune lipid extract. Bars without a common letter are significantly different $(P<.05)$ using one-way ANOVA and Tukey's pairwise comparison. Values are means $\pm \operatorname{SEM}(n=4-5)$.

\subsection{Repression of the expression of proinflammatory mediators by N. commune lipid extract} Despite that health benefits from $N$. commune have been recognized in Chinese medicine, there are very few studies in the scientific literature that have addressed this issue. To investigate whether lipid extract from $N$. commune could decrease the expression of proinflammatory mediators in RAW 264.7 macrophages, we incubated the cells with increasing concentrations of $N$. commune lipid extract $(0-100 \mu \mathrm{g} / \mathrm{mL})$ for 12 hours. Subsequently, the cells were activated with LPS (100 ng/mL), a component of gram-negative bacterial cell wall, for an additional 18 hours. $N$. commune lipid extract reduced mRNA abundance of proinflammatory mediators, including TNF- $\alpha$, COX-2, IL- $1 \beta$, IL-6, and iNOS, in a dosedependent manner (Fig. 3A). Consistent with mRNA levels, secretion of TNF- $\alpha$ and IL-1 $\beta$ into culture medium was also significantly reduced by N. commune lipid extract (Fig. 3B). $N$. commune lipid extract appears to contain bioactive compounds that can antagonize the production of LPS-induced proinflammatory mediators. 


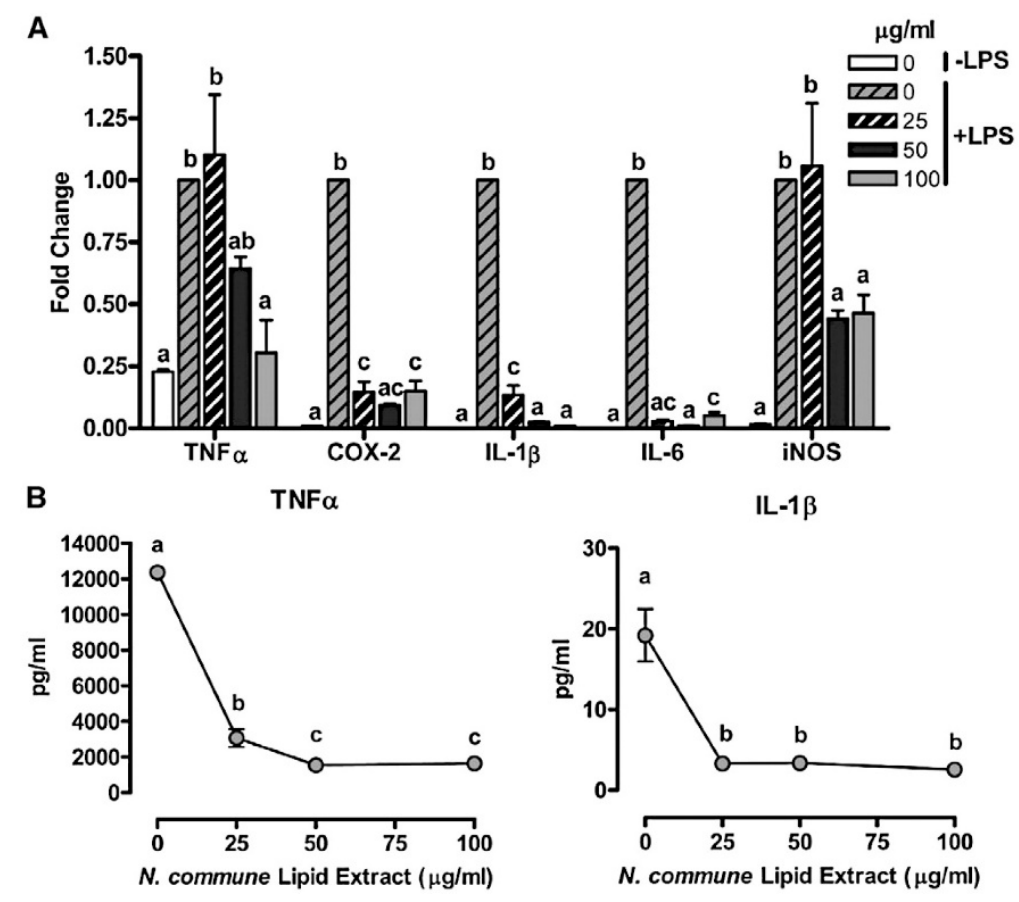

Figure 3. N. commune lipid extract inhibited the production of proinflammatory mediators in LPS-activated RAW 264.7 macrophages. Cells were incubated with increasing concentrations of $N$. commune lipid extract $(0-100 \mu \mathrm{g} / \mathrm{mL})$ for 12 hours, and LPS (100 ng/mL) was added to activate macrophages for 18 hours. (A) Real-time PCR analysis for mRNA expression of proinflammatory genes. Data are expressed as fold change relative to control treated with LPS. Bars without a common letter are significantly different $(P<.05)$. Values are means \pm SEM $(n=4-5)$. (B) Cytokine concentrations. TNF- $\alpha$ and IL- $1 \beta$ concentrations in the medium collected after the incubation with $N$. commune lipid extract and LPS were determined by ELISA. Data without a common letter are significantly different $(P<.05)$ using one-way ANOVA and Tukey's pairwise comparison. Values are means $\pm \operatorname{SEM}(n=6)$.

\subsection{Effect of fatty acid mixture on the expression of proinflammatory mediators}

Palmitoleic acid, linoleic acid, and linolenic acid, which consist of approximately $75 \%$ of total fatty acids in N. commune lipid extract, inhibited the expression of proinflammatory genes in RAW 264.7 macrophages, whereas palmitic acid increased the expression of those genes (unpublished data). To address whether the unsaturated fatty acids in N. commune lipid extract play a major role in the reduced production of proinflammatory mediators by $N$. commune, we formulated a fatty acid mixture that reflects the same fatty acid composition of N. commune lipid extract. TLC-densitometry analysis showed that the N. commune lipid extract contains approximately $15 \%$ fatty acids (Fig. 1 ). Therefore, we incubated RAW 264.7 macrophages with $N$. commune lipid extract $(100 \mu \mathrm{g} / \mathrm{mL})$ or $15 \mu \mathrm{g} / \mathrm{mL}$ of a fatty acid mixture, which contains the same amount and composition of fatty acids as $100 \mu \mathrm{g} / \mathrm{mL}$ of the lipid extract, for 12 hours. Subsequently, the cells were activated by LPS for 18 hours. LPS drastically induced mRNA expression of TNF- $\alpha$ and COX-2 in control groups, whereas N. commune lipid extract inhibited the induction of TNF- $\alpha$ and COX-2 expression (Fig. 4). 
Although the addition of $15 \%$ of fatty acid mixture significantly reduced the mRNA abundance of both genes compared with LPS-treated control, the degree of repression was less than that of $N$. commune lipid extract. The data suggest that in addition to unsaturated fatty acids, other bioactive compounds with an anti-inflammatory role are present in N. commune lipid extract that may inhibit the production of proinflammatory mediators in RAW 264.7 macrophages.
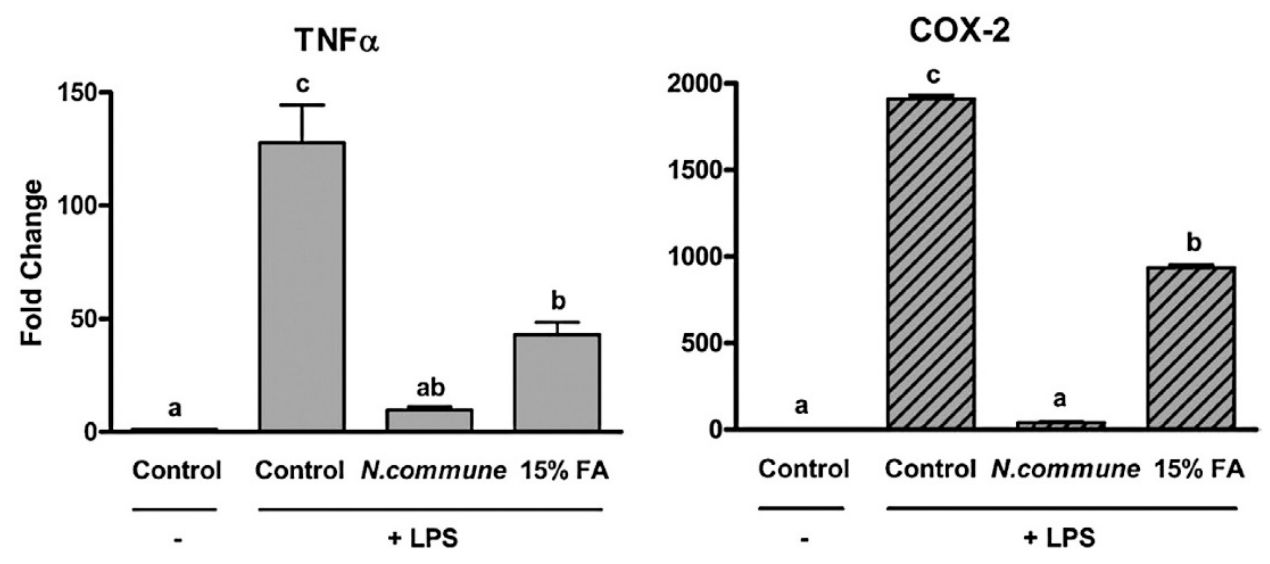

Figure 4. N. commune lipid extract contains bioactive compounds with an anti-inflammatory property. RAW 264.7 macrophages were incubated with $N$. commune lipid extract (100 $\mu \mathrm{g} / \mathrm{mL})$ or fatty acid mixture $(15 \mu \mathrm{g} / \mathrm{mL})$ for 12 hours, after which cells were activated by LPS (100 ng/mL) for 18 hours. Real-time PCR analysis was performed to measure mRNA abundance of TNF- $\alpha$ and COX-2. Data are a representative of 2 separate experiments and expressed as fold change relative to control without LPS treatment. Bars without a common letter are significantly different $(P<.05)$ using one-way ANOVA and Tukey's pairwise comparison. Values are means $\pm \operatorname{SEM}(n=3)$.

\subsection{Inhibition of $N F-\kappa B$ p65 DNA binding activity by $\mathrm{N}$. commune lipid extract}

To address whether $N$. commune lipid extract inhibits the production of proinflammatory mediators by blocking the NF- $\kappa \mathrm{B}$ signaling pathway, we measured p65 DNA-binding activity in RAW 264.7 macrophages after the incubation with $100 \mu \mathrm{g} / \mathrm{mL}$ of $N$. commune lipid extract. Control experiments were run simultaneously for validity of assay using a positive control and wild-type and mutant oligos provided by the manufacturer (data not shown). In the absence of $N$. commune lipid extract, LPS significantly increased p65 DNA binding activity (Fig. 5). However, the addition of N. commune lipid extract inhibited LPS-induced $\mathrm{NF}-\kappa \mathrm{B}$ activation, indicating that the anti-inflammatory role of $N$. commune lipid extract is mediated, at least in part, through its inhibition of NF- $\kappa$ B activation. 


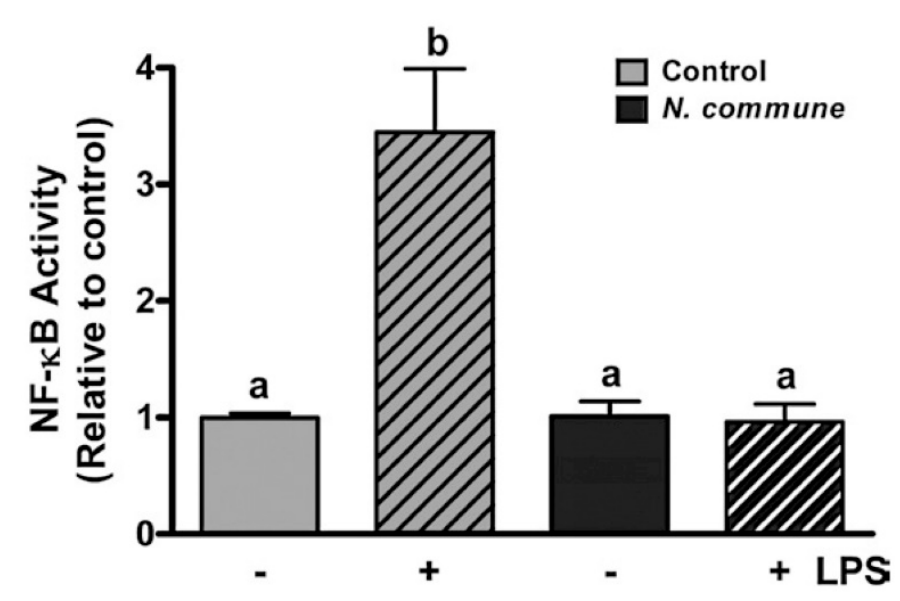

Figure 5. NF- $\kappa$ B p65 DNA-binding activity was inhibited by $N$. commune lipid extract. RAW 264.7 macrophages were incubated with 0 or $100 \mu \mathrm{g} / \mathrm{mL}$ of $N$. commune lipid extract for 12 hours, after which they were activated by LPS (100 ng/mL) for 1 hour. Nuclear extract was prepared, and p65 DNA-binding activity was measured using TransAM NF- $\kappa \mathrm{B}$ p65 assay kit. Data are expressed as activity relative to control without LPS. Bars without a common letter are significantly different $(P<.001)$ using one-way ANOVA and Tukey's pairwise comparison. Values are means $\pm \operatorname{SEM}(n=6)$.

\section{Discussion}

In this study, we investigated whether N. commune exerts an anti-inflammatory function and, if so, the mechanisms of action underlying such a function. We used cultivated $N$. commune, which is free of contamination of toxins and heavy metals and is under tight quality control during culture. Lipid extract from cultivated N. commune reduced the production of proinflammatory mediators by inhibiting, at least in part, the activation of NF- $\kappa \mathrm{B}$ pathway in RAW 264.7 macrophages.

$N$. commune lipid extract contains pigments, free fatty acids, triacylglycerol, vitamins, and wax and steryl esters as demonstrated by TLC-densitometry analysis. Fatty acids have been implicated in modulating inflammatory responses [25] and they comprise approximately $15 \%$ of the total N. commune lipid extract. In addition, as approximately $75 \%$ of fatty acids in N. commune lipid extract are unsaturated fatty acids with a potential anti-inflammatory property, we reasoned that fatty acids could be the major bioactive components in N. commune lipid extract. As we expected, the fatty acid mixture that was constructed to reflect the same fatty acid composition in the N. commune lipid extract reduced the expression of proinflammatory mediators. The extent of the reduction, however, was less than that of $N$. commune lipid extract, indicating there are other bioactive components in the lipid extract that possess anti-inflammatory properties.

Various bioactive compounds from blue-green algae have been reported [26]. A wellknown bioactive compound found in various blue-green algae is C-phycocyanin, a watersoluble protein-bound pigment that accounts for more than $20 \%$ of algal dry weight [27]. In animal models of inflammation, such as rats and mice treated with arachidonic acid on 
ears and carrageenan injection in paws, C-phycocyanin reduced inflammation, and this effect was attributed to its antioxidative and oxygen free radical scavenging properties $[7,8]$. However, as C-phycocyanin is water-soluble, other unidentified bioactive components bearing an anti-inflammatory property are presumed to be present in N. commune lipid extract. It needs to be determined whether cultivated $N$. commune contains a high content of C-phycocyanin, similar to other blue-green algae. Bluegreen algae in general contain a high amount of carotenoids and the antioxidant properties of $\beta$-carotene could play a role in preventing inflammatory response. Studies have shown an inverse relationship between plasma $\beta$-carotene levels and makers of inflammation, such as sialic acid and C-reactive protein $[28,29]$. In LPS-stimulated RAW 264.7 macrophages, $\beta$-carotene prevented inflammatory gene expression by inhibiting NF- $\kappa \mathrm{B}$ activation [30]. N. commune used in the present study contains approximately $70 \mathrm{mg} / 100 \mathrm{~g}$ (dry weight) of $\beta$-carotene, which is more than that of dried carrots (approximately $51 \mathrm{mg} / 100 \mathrm{~g}$, US Department of Agriculture database). It is possible that the anti-inflammatory effect of $N$. commune lipid extract is conferred, in part, by its high $\beta$-carotene content. N. commune has been used for treating night blindness in Chinese medicine, and this effect could be attributed to high $\beta$-carotene content. Further chemical analysis for composition of $N$. commune lipid extract is necessary to identify additional bioactive compounds with an anti-inflammatory property.

Inflammatory response to LPS is primarily mediated through the toll-like receptor 4 (TLR-4) pathway [31,32]. Binding of LPS to TLR-4 and its coreceptors, such as cluster of differentiation 14 (CD14) and MD-2, triggers downstream signaling cascades, which lead to the activation of NF- $\kappa$ B. Recently, Macagno et al. [9] reported that LPS-like molecule extracted from a fresh-water cyanobacterium Oscillatoria planktothrix FP1 strongly inhibited the expression of proinflammatory cytokines in human dendritic cells as well as endotoxin shock in mice. In the study, authors showed that the LPS-like molecule from the cyanobacterium competes with LPS for binding to the TLR-4-MD2 receptor complex, possibly blocking LPS-induced proinflammatory signaling cascades. The nature of the repressive effect of $N$. commune lipid extract on the production of proinflammatory mediators needs to be further examined. NF- $\kappa \mathrm{B}$ is a dimeric transcription factor that consists of p65 (RelA), c-Rel, RelB, p50/105, and p52/100 [33]. Activity of NF- $\kappa$ B can be increased by phorbor esters, proinflammatory cytokines such as TNF- $\alpha$ and IL-1, and LPS [34]. In an unstimulated state, $\mathrm{NF}-\kappa \mathrm{B}$ is present in the cytoplasm bound with inhibitors of NF- $\kappa \mathrm{B}$ (I $\kappa \mathrm{B}) \alpha / \beta$, which masks the nuclear localization sequence of p65 [35]. In response to inflammatory stimulation, $\mathrm{I} \kappa \mathrm{B} \alpha / \beta$ are phosphorylated, which are destined for ubiquitination and subsequent degradation by proteasomes [36-40]. Consequently, NF- $\kappa$ B becomes free to translocate from the cytoplasm to the nucleus where it binds to the $\kappa \mathrm{B}$ element of target genes participating in the inflammatory and immune processes [41]. As transcription of various genes that encode proinflammatory cytokines, chemokines, and other effectors of the innate immune response is increased upon the activation of NF- $\kappa \mathrm{B}, \mathrm{NF}-\kappa \mathrm{B}$ has been a target for the treatment of inflammatory diseases [42]. Inhibition of NF- $\kappa$ B activation and the consequent repression of various proinflammatory mediators by N. commune lipid extract in our study suggest its potential to be used as an anti-inflammatory agent. 
In conclusion, we have demonstrated for the first time that a lipid extract of a blue-green alga, $N$. commune, represses the expression of several genes involved in the proinflammatory response to inflammatory stimuli by the inhibition of signaling cascades leading to the activation of NF- $\kappa \mathrm{B}$. Because NF- $\kappa \mathrm{B}$ regulates various genes involved in the inflammatory responses of cells, it has been a target for the treatment of acute or chronic inflammatory diseases such as sepsis, Crohn's disease, rheumatoid arthritis, and atherosclerosis $[43,44]$. Therefore, our study provides strong scientific evidence for $N$. commune to be developed as a new health-enhancing nutritional food for the prevention and treatment of chronic inflammatory diseases and further advances our knowledge in molecular nutrition by elucidating pathways for the functional food at a molecular level.

Acknowledgments - Authors thank Dave Giraud for HPLC analysis of carotenoid contents. This work was supported by National Science Foundation-EPSCoR grant EPS-0346476 and the Hatch Act (JY Lee), by University of Nebraska-Lincoln Agricultural Research Division Honors Student Undergraduate Research (KR Blobaum), and by US Department of Agriculture SBIR grant 2003-00102 (F Lu). This study is a contribution of the University of Nebraska Agricultural Research Division.

\section{References}

[1] Guslandi M. Nitric oxide and inflammatory bowel diseases. Eur J Clin Invest 1998;28:904-7.

[2] Simons RK, Junger WG, Loomis WH, Hoyt DB. Acute lung injury in endotoxemic rats is associated with sustained circulating IL-6 levels and intrapulmonary CINC activity and neutrophil recruitment-role of circulating TNF-alpha and IL-beta? Shock 1996;6:39-45.

[3] Jugdutt BI. Cyclooxygenase inhibition and adverse remodeling during healing after myocardial infarction. Circulation 2007;115:288-91.

[4] Stanier RY, Cohen-Bazire G. Phototrophic prokaryotes: the cyanobacteria. Annu Rev Microbiol 1977;31:225-74.

[5] Prinsep MR, Thomson RA, West ML, Wylie BL. Tolypodiol, an antiinflammatory diterpenoid from the cyanobacterium Tolypothrix nodosa. J Nat Prod 1996;59:786-8.

[6] Oufdou K, Mezrioui N, Oudra B, Loudiki M, Barakate M, Sbiyyaa B. Bioactive compounds from Pseudanabaena species (Cyanobacteria). Microbios 2001;106(Suppl 1):21-9.

[7] Romay C, Ledon N, Gonzalez R. Further studies on anti-inflammatory activity of phycocyanin in some animal models of inflammation. Inflamm Res 1998;47:334-8.

[8] Romay C, Armesto J, Remirez D, Gonzalez R, Ledon N, Garcia I. Antioxidant and antiinflammatory properties of C-phycocyanin from blue-green algae. Inflamm Res 1998;47:36-41.

[9] Macagno A, Molteni M, Rinaldi A, Bertoni F, Lanzavecchia A, Rossetti C, et al. A cyanobacterial LPS antagonist prevents endotoxin shock and blocks sustained TLR4 stimulation required for cytokine expression. J Exp Med 2006;203:1481-92.

[10] Gilroy DJ, Kauffman KW, Hall RA, Huang X, Chu FS. Assessing potential health risks from microcystin toxins in blue-green algae dietary supplements. Environ Health Perspect 2000;108: 435-9.

[11] Potts M, Olie JJ, Nickels JS, Parsons J, White DC. Variation in phospholipid ester-linked fatty acids and carotenoids of desiccated Nostoc commune (Cyanobacteria) from different geographic locations. Appl Environ Microbiol 1987;53:4-9. 
[12] Cao K. Chinese studies on the edible blue-green algae, Nostoc flagelliforme: a review. J Appl Phycol 1998;10:37-49.

[13] Qui B, Liu J, Liu Z, Liu S. Distribution and ecology of the edible cyanobacterium Ge-Xian-Mi (Nostoc) in rice fields of Hefeng County in China. J Appl Phycol 2002;14:423-9.

[14] Hori K, Ishibashi G, Okita T. Hypocholesterolemic effect of blue-green alga, ishikurage (Nostoc commune) in rats fed atherogenic diet. Plant Foods Hum Nutr 1994;45:63-70.

[15] Esser MT, Mori T, Mondor I, Sattentau QJ, Dey B, Berger EA, et al. Cyanovirin-N binds to gp120 to interfere with $\mathrm{CD} 4$-dependent human immunodeficiency virus type 1 virion binding, fusion, and infectivity but does not affect the CD4 binding site on gp120 or soluble CD4-induced conformational changes in gp120. J Virol 1999;73:4360-71.

[16] Knubel G, Larsen LK, Moore RE, Levine IA, Patterson GM. Cytotoxic, antiviral indolocarbazoles from a blue-green alga belonging to the Nostocaceae. J Antibiot (Tokyo) 1990;43:1236-9.

[17] Smith CD, Zhang X, Mooberry SL, Patterson GM, Moore RE. Cryptophycin: a new antimicrotubule agent active against drug-resistant cells. Cancer Res 1994;54:3779-84.

[18] Vadiraja BB, Gaikwad NW, Madyastha KM. Hepatoprotective effect of C-phycocyanin: protection for carbon tetrachloride and R-(+)-pulegone-mediated hepatotoxicty in rats. Biochem Biophys Res Commun 1998;249:428-31.

[19] Bhat VB, Madyastha KM. C-phycocyanin: a potent peroxyl radical scavenger in vivo and in vitro. Biochem Biophys Res Commun 2000;275:20-5.

[20] Bligh EG, Dyer WJ. A rapid method of total lipid extraction and purification. Can J Med Sci 1959;37:911-7.

[21] Carr TP, Weller CL, Schlegel VL, Cuppett SL, Guderian Jr DM, Johnson KR. Grain sorghum lipid extract reduces cholesterol absorption and plasma non-HDL cholesterol concentration in hamsters. J Nutr 2005;135:2236-40.

[22] Metcalfe LD, Schmitz AA, Pelka JR. Rapid preparation of fatty acid esters from lipids for gas chromatgraphic analysis. Anal Chem 1966;38:514-5.

[23] Hart DJ, Scott KJ. Development and evaluation of an HPLC method for the analysis of carotenoids in foods, and the measurement of the carotenoid content of vegetables and fruits commonly consumed in the UK. Food Chem 1995;54:101-11.

[24] Livak KJ, Schmittgen TD. Analysis of relative gene expression data using real-time quantitative PCR and the $2(-\Delta \Delta \mathrm{C}(\mathrm{T}))$ method. Methods 2001;25:402-8.

[25] Calder PC. n-3 Polyunsaturated fatty acids, inflammation, and inflammatory diseases. Am J Clin Nutr 2006;83:S1505-19.

[26] Patterson GML, Larsen LK, Moore RE. Bioactive natural products from blue-green algae. J Appl Phycol 1994;6:151-7.

[27] Richmond A. Large scale microalgal culture and applications. In: Chapman R, editor. Progress in phycological research. Bristol: Biopress Ltd; 1990. p. 8.

[28] Iribarren C, Folsom AR, Jacobs Jr DR, Gross MD, Eckfeldt JH, Kritchevsky SB. Patterns of covariation of serum $\beta$-carotene and $\alpha$-tocopherol in middle-aged adults: the Atherosclerosis Risk in Communities (ARIC) Study. Nutr Metab Cardiovasc Dis 1997; 7:445-58.

[29] Boosalis MG, Snowdon DA, Tully CL, Gross MD. Acute phase response and plasma carotenoid concentrations in older women: findings from the nun study. Nutrition 1996;12:475-8.

[30] Bai SK, Lee SJ, Na HJ, Ha KS, Han JA, Lee H, et al. $\beta$-Carotene inhibits inflammatory gene expression in lipopolysaccharide-stimulated macrophages by suppressing redox-based NF- $\kappa \mathrm{B}$ activation. Exp Mol Med 2005;37:323-34. 
[31] Fitzgerald KA, Rowe DC, Golenbock DT. Endotoxin recognition and signal transduction by the TLR4/MD2-complex. Microbes Infect 2004;6:1361-7.

[32] Kawai T, Akira S. TLR signaling. Cell Death Differ 2006;13:816-25.

[33] Pasparakis M, Luedde T, Schmidt-Supprian M. Dissection of the NF- $\kappa B$ signalling cascade in transgenic and knockout mice. Cell Death Differ 2006;13:861-72.

[34] Baldwin AS. The NF-B and IB proteins: new discoveries and insights. Annu Rev Immunol 1996; 14:649-81.

[35] Hayden MS, Ghosh S. Signaling to NF- $\kappa$ B. Genes Dev 2004;18: 2195-224.

[36] DiDonato J, Mercurio F, Rosette C,Wu-Li J, Suyang H, Ghosh S, et al. Mapping of the inducible I KB phosphorylation sites that signal its ubiquitination and degradation. Mol Cell Biol 1996;16: 1295-304.

[37] Chen Z, Hagler J, Palombella VJ, Melandri F, Scherer D, Ballard D, et al. Signal-induced sitespecific phosphorylation targets I $\kappa$ B $\alpha$ to the ubiquitin-proteasome pathway. Genes Dev 1995;9:1586-97.

[38] Brown K, Gerstberger S, Carlson L, Franzoso G, Siebenlist U. Control of I $\kappa$ B- $\alpha$ proteolysis by site-specific, signal-induced phosphorylation. Science 1995;267:1485-8.

[39] DiDonato JA, Mercurio F, Karin M. Phosphorylation of $\mathrm{I} \kappa \mathrm{B} \alpha$ precedes but is not sufficient for its dissociation from NF- $\kappa$ B. Mol Cell Biol 1995;15:1302-11.

[40] Alkalay I, Yaron A, Hatzubai A, Jung S, Avraham A, Gerlitz O, et al. In vivo stimulation of I $\mathcal{K}$ B phosphorylation is not sufficient to activate NFK B. Mol Cell Biol 1995;15:1294-301.

[41] Karin M. The beginning of the end: I $\kappa B$ kinase (IKK) and NF- $\kappa B$ activation. J Biol Chem 1999; 274:27339-42.

[42] Zuany-Amorim C, Hastewell J, Walker C. Toll-like receptors as potential therapeutic targets for multiple diseases. Nat Rev Drug Discov 2002;1:797-807.

[43] Baeuerle PA, Henkel T. Function and activation of NF- $\kappa$ B in the immune system. Annu Rev Immunol 1994;12:141-79.

[44] Brand K, Page S, Walli AK, Neumeier D, Baeuerle PA. Role of nuclear factor- $\kappa$ B in atherogenesis. Exp Physiol 1997;82:297-304. 